\title{
Accelerated first-in-human clinical trial of EIDD-2801/MK-4482 (molnupiravir), a ribonucleoside analog with potent antiviral activity against SARS-CoV-2
}

Wendy Holman', Wayne Holman', Stacy McIntosh', Wendy Painter ${ }^{2}$, George Painter $^{2}$, Jim Bush ${ }^{3}$ and Oren Cohen ${ }^{4^{*}}$ (1)

\begin{abstract}
A recently published article described the safety, tolerability, and pharmacokinetic profile of molnupiravir (Painter et al. 2021), a novel antiviral agent with potent activity against severe acute respiratory syndrome coronavirus 2 (SARS-CoV-2), the causative agent of coronavirus disease 2019 (COVID-19). Here, we report an unprecedented collaboration between sponsor, contract research organization (CRO), and regulatory authorities that enabled accelerated generation of these phase I data, including administration of the first-in-human (FIH) dose of molnupiravir within 5 days of receiving regulatory approval in the United Kingdom (UK). Single and multiple ascending dose (SAD and MAD, respectively) cohorts were dosed in randomized, double-blind, and placebocontrolled fashion, with a 6:2 active-to-placebo ratio in each cohort. A food-effect (FE) cohort included 10 subjects who were randomized to receive drug in the fasted or fed state followed by the fed or fasted state to complete a fed and fasted sequence for each subject. Dose escalation decisions were accelerated and MAD cohorts were initiated prior to completion of all SAD cohorts with the provision that the total daily dose in a MAD cohort would not exceed a dose proven to be safe and well-tolerated in a SAD cohort. Dosing in healthy volunteers was completed for eight single ascending dose (SAD) cohorts, seven multiple ascending dose (MAD) cohorts, and one food-effect (FE) cohort within approximately 16 weeks of initial protocol submission to the Research Ethics Committee (REC) and Medicines and Healthcare products Regulatory Agency (MHRA). Working to standard industry timelines, the FlH study would have taken approximately 46 weeks to complete and 33 weeks to enable phase 2 dosing. Data from this study supported submission of a phase $2 / 3$ clinical trial protocol to the US Food and Drug Administration (FDA) within 8 weeks of initial protocol submission, with FDA comments permitting phase 2 study initiation within two additional weeks. In the setting of a global pandemic, this model of collaboration allows for accelerated generation of clinical data compared to standard processes, without compromising safety.
\end{abstract}

Keywords: COVID-19, SARS-CoV-2, Antiviral therapy, Ribonucleoside analog, Phase I, Accelerated start-up

\footnotetext{
* Correspondence: oren.cohen@covance.com

${ }^{4}$ Covance, Inc., 6 Drive, Research Triangle Park, Moore, NC 27709, USA

Full list of author information is available at the end of the article
}

(c) The Author(s). 2021 Open Access This article is licensed under a Creative Commons Attribution 4.0 International License, which permits use, sharing, adaptation, distribution and reproduction in any medium or format, as long as you give appropriate credit to the original author(s) and the source, provide a link to the Creative Commons licence, and indicate if changes were made. The images or other third party material in this article are included in the article's Creative Commons licence, unless indicated otherwise in a credit line to the material. If material is not included in the article's Creative Commons licence and your intended use is not permitted by statutory regulation or exceeds the permitted use, you will need to obtain permission directly from the copyright holder. To view a copy of this licence, visit http://creativecommons.org/licenses/by/4.0/ The Creative Commons Public Domain Dedication waiver (http://creativecommons.org/publicdomain/zero/1.0/) applies to the data made available in this article, unless otherwise stated in a credit line to the data. 


\section{Background}

Coronavirus disease 2019 (COVID-19) was initially described as a new infectious respiratory illness in late 2019 [1-7]. A new pathogenic coronavirus, severe acute respiratory syndrome coronavirus 2 (SARS-CoV-2), was quickly shown to be the causative agent of COVID-19 [1, 5-7]. Clinical manifestations of COVID-19 range from asymptomatic infection to pneumonia to acute respiratory distress syndrome (ARDS), cytokine storm, coagulopathy, thrombosis, and a number of other extrapulmonary syndromes $[4,8-10]$. In some instances, an otherwise benign disease course can very rapidly deteriorate during the second week of illness, often requiring supplemental oxygen therapy and intensive care $[4,9]$. Although aberrant immune responses may drive some COVID-19 pathology, it is clear that a high level of viral replication early during the course of disease is associated with disease severity $[11,12]$. Thus, there is an urgent need for a potent antiviral agent that does not require parenteral administration and, therefore, can be given early in the course of disease. Given the current understanding of COVID-19 pathogenesis, such a strategy involving early potent antiviral therapy should have a high probability of success.

Molnupiravir is the orally bioavailable $5^{\prime}$-isobutyrate prodrug of a direct-acting antiviral ribonucleoside analog, $\beta$-D-N4-hydroxycytidine, or EIDD-1931. Molnupiravir is cleaved in the plasma to liberate EIDD-1931. EIDD-1931 is phosphorylated intracellularly to its corresponding 5 '-triphosphate, the active antiviral agent, by host kinases [13, 14]. EIDD-1931 inhibits replication of multiple RNA viruses, and its antiviral activity was verified in animal models of influenza, various coronaviruses, respiratory syncytial virus (RSV), Venezuelan equine encephalitis virus (VEEV), Chikungunya, and Ebola virus infection [15-18].

Similar to other nucleoside analog antiviral agents, EIDD-1931 5' -triphosphate acts as a competitive alternative substrate for the virally encoded RNA-directed RNA polymerases ( $\mathrm{RdRp}$ ) and is incorporated into nascent chain RNA. Since the N4-hydroxycytosine base of EIDD-1931 can tautomerize, EIDD-1931 can substitute for either cytidine or uridine and pair with either guanosine or adenosine, respectively, thereby disrupting the fidelity of viral genomic replication [19]. This results in an accumulation of mutations that increases with each cycle of viral replication. The process whereby the mutation rate is increased by exposure to a drug is referred to as viral decay acceleration and results in viral ablation [20]. This mechanism of action for EIDD-1931 has been validated through demonstration of activity against Middle East respiratory syndrome coronavirus (MERS-CoV), VEEV, and influenza A virus (IAV) $[15,18,21,22]$.
When SARS-CoV-2 emerged as a global health threat in late 2019, an Investigational New Drug (IND) application for molnupiravir was being prepared for the treatment of IAV. At that time, there were in vitro data demonstrating that EIDD-1931 was active against other pathogenic coronaviruses [15]. It was quickly demonstrated that EIDD-1931 had potent antiviral activity against SARS-CoV-2 in a human lung epithelial cell line and against a clinical isolate of SARS-CoV-2 (2019-nCoV/USA-WA1/2020) in African green monkey kidney (Vero) cells [22]. In primary human airway epithelial cell cultures, which recapitulate the architecture and cellular complexity of the conducting airway, EIDD-1931 also demonstrated potent, dose-dependent in vitro antiviral activity against severe acute respiratory syndrome coronavirus (SARSCoV), MERS-CoV, and SARS-CoV-2 [22].

Although nucleoside analogs are important components of modern antiviral therapy, their activity can be markedly reduced by a single or small number of viral mutations. The structural motifs of the RdRp are highly conserved among coronaviruses, including SARS-CoV-2. Resistance to another broad spectrum ribonucleoside analog, remdesivir, is mediated by mutations in $\mathrm{RdRp}$ residues 480 (F480L) and 557 (V557L) in SARS-CoV and in a murine coronavirus, mouse hepatitis virus (MHV) [23]. Of note, these two remdesivir resistance mutations, alone or in combination, actually conferred increased sensitivity to inhibition by EIDD-1931 [22].

In a mouse model of SARS-CoV infection, administration of molnupiravir beginning $2 \mathrm{~h}$ before or 12 or $24 \mathrm{~h}$ after infection demonstrated robust antiviral activity and amelioration of disease (including parameters such as body weight, viral load in lung tissue, and lung hemorrhage), over a 5-day observation period [22].

The phase I clinical trial data describing the safety, tolerability, and pharmacokinetic profile of molnupiravir were recently published [24]. This paper describes how these data were generated along a significantly accelerated timeline.

\section{Rapid initiation of a clinical research program to study molnupiravir as a treatment for COVID-19 Start-up activities}

Following an initial call with the sponsor, the contract research organization (CRO) immediately assembled a project team composed of clinical pharmacology, operations, medical, pharmacy, bioanalytical, data management, biostatistics, medical writing, project management, pharmacokinetics, and regulatory staff (project day 1). A joint sponsor-CRO project team developed an expedited phase 1 single ascending dose (SAD) and food-effect (FE) trial design, which included a placeholder for the later addition of multiple ascending dose (MAD) 
cohorts. This approach facilitated rapid startup and reduced the time to acquire MAD cohort data at physiologically relevant doses (based on animal model data [17]) to 40 calendar days from project initiation.

In order to maximize the likelihood of completing the phase 1 study without interruption due to possible COVID-19-related closure of investigative sites, regulatory submissions in the USA and UK were pursued in parallel. In the USA, a recently submitted molnupiravir IND for the treatment of IAV provided the foundation for submission of preliminary data on the drug's activity against pathogenic coronaviruses. The Food and Drug Administration (FDA) expedited the conduct of a PreIND Meeting and review of the SARS-CoV-2 IND (cross-referencing the IAV IND). The IAV IND content plus the SARS-CoV-2 data were the basis for submission packages in the UK. An Expert Working Group for COVID-19 was established by the UK Commission on Human Medicines, and the Medicines and Healthcare products Regulatory Agency (MHRA) published a guidance on Clinical Trial Applications (CTAs) for COVID19 products, which specified the procedures to supply rapid scientific advice, review, and approval for potential COVID-19 treatments [25]. MHRA confirmed that the CTA should include all the usual components (i.e., Investigator's Brochure, Study Protocol and Investigational Medicinal Product Dossier), but that review of final draft documents would occur on a rolling basis. Review comments were provided by the MHRA in real-time, permitting the project team to make requested changes prior to formal CTA submission. The MHRA further advised that Research Ethics Committee (REC) submission should not proceed in the usual fashion, via the Combined Ways of Working pathway (which allows consecutive regulatory and ethics review from a single application), but rather a request for expedited review should be made through the Health Research Authority (HRA) Director of Approvals Service. With regard to the protocol, the MHRA advised that the SAD design should be fully defined in the initial submission, but that subsequent components (e.g., MAD cohorts) could be referenced as placeholders for tailoring at a later date. Protocol amendments received similar expedited review by both MHRA and the REC.

Over the first 11 days of the project, all MHRA regulatory submission documents were completed and the SAD study protocol was finalized, culminating in submission to the REC on day 12 of the project. The REC convened the following day, with the principal investigator (PI) in attendance by telephone. Provisional REC approval was granted on day 16 of the project. On that same day, formal CTA submission was achieved. Full REC approval was received on day 18 and CTA approval on day 19 of the project, respectively. This enabled the screening of healthy volunteers to start on day 20 and the first human dose to be administered on day 23 of the project, respectively.

In parallel, the CRO pharmacy prepared for supply of the investigational medicinal product in accordance with Good Manufacturing Practices. In order to further expedite study start-up, a decision was made to dose SAD cohorts with a powder-in-bottle preparation which was used for extemporaneous preparation of a solution for oral administration; a capsule preparation was later introduced for MAD cohorts through CTA amendment. Active Pharmaceutical Ingredient (API) was received by the CRO pharmacy on day 17 of the project. On day 18 , API samples were sent to a contract laboratory for confirmatory identification (ID) testing. ID testing and the technical batch run were both completed on day 19 . Pharmacy setup activities, which routinely require two to three months to perform, were completed within seven calendar days. Bioanalytical setup was also completed very rapidly, with plasma method development work and core plasma validation experiments completed in 2 weeks, rather than the usual 5 weeks.

\section{Protocol design}

The initial protocol design (CTIN NCT04392219) included two parts: a randomized, double-blind, placebo-controlled SAD study of three cohorts (part 1) and a FE cohort (part 2). The protocol was written to include up to four additional cohorts in part 1 and one additional cohort in part 2. SAD study cohorts included eight subjects each randomized 6:2 to molnupiravir and placebo, respectively. The initial SAD cohort included dosing of two sentinel subjects, each one assigned to either molnupiravir or placebo. The safety results for the sentinel group were reviewed 24 $h$ post-dose, prior to dosing the remainder of the subjects in cohort 1 . Following dosing on study day 1 , subjects remained in the clinic through completion of study assessments on study day 4. Subjects returned to the clinic on study day 9 for assessments and study day 15 for the end of study (EOS) visit.

For part 2, the effect of food on molnupiravir pharmacokinetic (PK) parameters was examined in a single cohort of 10 healthy volunteers. Five subjects were randomized to receive drug in the fed then fasted state (sequence 1), and five subjects were randomized to receive drug in the fasted then fed state (sequence 2), with an intervening washout period in the middle of each sequence. Treatment was administered in open-label fashion. In each sequence, subjects were discharged from the clinic on the fourth day after dosing and returned to the clinic on the ninth day after dosing for blood draw and assessments. Subjects returned on the fourteenth day after dosing 
for check-in to begin the second dosing sequence (fasting if sequence 1 was in the fed state, and fed if sequence 1 was in the fasting state) on study day 15 .

Upon initiation of part 1 (day 23), a protocol amendment to add a MAD component (part 3) was submitted to the MHRA and REC on days 23 and 24, respectively. Again, a final draft protocol was informally submitted to MHRA and comments were provided in real-time prior to formal submission of the substantial amendment. This enabled MHRA and REC approvals 2 days after formal submission, on days 25 and 26, respectively. A comparable IND amendment was submitted in parallel to FDA for US sites as a back-up. Part 3 was a randomized, double-blind, placebo-controlled MAD study with three initial cohorts and the option to include up to four additional cohorts. Cohorts in the MAD study included eight subjects each, randomized 6:2 to molnupiravir and placebo, respectively. Subjects were dosed twice daily on study days $1-5$ and received a further single dose on the morning of study day 6 . Subjects remained in the clinic through completion of study assessments on study day 9. Subjects returned to the clinic on study day 15 for assessments and on study day 20 for the end of study visit. The protocol allowed part 3 to run in staggered parallel fashion with part 1 , provided that the total daily dose administered in part 3 did not exceed a dose already shown to be safe and well-tolerated in part 1 .

In parts 1 (SAD) and 3 (MAD), dose escalation proceeded based upon satisfactory review by the PI, Medical Monitor, and sponsor of the blinded safety and tolerability data collected up to $72 \mathrm{~h}$ post-dose for a preceding dose cohort. The 72 -h period was established based on the anticipated drug clearance. Plasma PK data from the lower dose levels were also reviewed as they became available. Dose escalation could only occur if data from a minimum of six subjects in total and a minimum of four subjects receiving molnupiravir were used to make the dose escalation decision. The dose levels tested in later MAD cohorts were selected so as not to increase the dose given by more than 3 -fold for predicted nonpharmacologically active dose levels and by 2 -fold for predicted pharmacologically active dose levels.

\section{Execution of the study}

The CRO volunteer recruitment team initiated contact with healthy volunteers registered in the CRO recruitment database with interest in participating in clinical trials before the CTA was approved. Volunteer screening commenced on day 20 of the project, 1 day after CTA approval was granted, and approximately 80 screening appointments were booked to occur between days $20-$ 22 of the project, over a public holiday weekend in the
UK. The first-in-human (FIH) dose was administered on day 23 of the project, just 15 business days after initial contact between sponsor and CRO. Rapid compilation of full safety information, including cohort characteristics, vital signs, electrocardiograms, complete blood counts with differential, clinical chemistry panels, urinalyses, and adverse event listings, was required while adhering to MHRA data quality standards. Dose escalation meetings were held on the day of cohort discharge irrespective of the day of the week. Subjects for a subsequent cohort were screened and brought to the clinic such that their dosing could proceed on the day following safety review, provided all dose escalation criteria were met.

The first three SAD cohorts were dosed on day 23/ 24 (sentinel group/main group), day 28 , and day 32 of the project. On day 32 of the project, the first MAD group was also dosed. Review of data from the first three cohorts of part 1 (SAD) indicated favorable PK and an absence of any safety or tolerability issues. Therefore, the decision was made to enroll cohorts at higher dose levels in part 1 than initially planned, in order to determine if any dose-limiting safety or tolerability issues would emerge. In addition, PK modeling suggested that higher clinical dose levels may be necessary; it was therefore necessary to study higher dose SAD cohorts to fully explore the therapeutic dose range. Following the SAD Cohort 3 dose escalation meeting, intensive communication with MHRA Medical Assessor enabled a meeting within days that included review of a summary rationale document, as well as the safety and PK data. The Medical Assessor then conferred with the Nonclinical Assessor and agreed with the proposed rationale. A protocol amendment was submitted to MHRA and the REC, and both approvals were received the following day (day 40 of the project), just 1 week from the time of the SAD Cohort 3 dose escalation meeting. Consistent with contingency plans noted above, submission to FDA occurred during the same week.

The second MAD cohort was dosed on day 41 of the project. SAD cohorts 4 and 5 were dosed on days 45 and 49 of the project, respectively. Review of these data provided the necessary safety clearance to dose the MAD cohorts 3 and 4 on days 50 and 59, respectively. The food effect study (part 2) was completed from days 3568 of the project.

The dose level administered in MAD cohort 3 was selected as the initial dose level to be administered to patients in phase 2. Molnupiravir was therefore positioned for phase 2 studies within approximately 8 weeks of protocol submission. On day 50 of the project, the sponsor submitted a phase $2 / 3$ protocol to FDA for a study of molnupiravir in patients with symptomatic COVID- 
19 not requiring hospitalization. FDA comments permitting study initiation were received on day 66 of the project, in turn enabling initiation of a pivotal trial following dose finding in the phase 2 studies.

SAD cohort 6, which received the increased maximum dose level allowed by the protocol, was dosed on day 60 of the project. Another meeting with the MHRA assessor transpired on day 73 of the project to provide rationale for a further increase to the maximum dose level allowed by the protocol, and a second substantial amendment was submitted to MHRA (day 87 ) and REC (day 88) with approvals received by day 95. MAD cohort 5 was successfully dosed on day 84 of the project, while waiting for MHRA and REC approval. Following approval, SAD cohorts 7 and 8 were dosed on days 100 and 114, respectively, and MAD cohorts 6 and 7 on days 105 and 121, respectively.

Dosing of the final cohort of subjects in the phase 1 program was completed approximately 16 weeks from the time of initial protocol submission for review by the regulatory authority and REC. Working to standard industry timelines, the FIH study would have normally taken approximately 46 weeks to complete and 33 weeks to enable phase 2 dosing (Fig. 1).

\section{Conclusion}

The speed with which this clinical program was accelerated is due to the extraordinary collaboration between sponsor, $\mathrm{CRO}$, and regulatory agencies, as well as the efficient study conduct made possible by the work of many individuals and by coordinated collaboration between stakeholders. Key success factors included alignment of all stakeholders with regard to a sense of urgency, execution of study elements in parallel wherever possible, acceleration of data processing and review, and contingency planning. Within eight weeks of phase 1 protocol submission, data were available that enabled dose selection for a phase 2 protocol for this promising new antiviral ribonucleoside analog. Had this study been conducted to standard industry timelines, it would have taken 8-9 months to generate the data necessary to enable a phase 2 study. Indeed, phase 2 studies have now commenced (ClinicalTrials.gov identifier NCT04405570 and NCT04405739), and study NCT04405570 completed in February, 2021. This case study demonstrates that urgent, coordinated efforts to support expedited study start-up and execution, including collaboration between sponsor, $\mathrm{CRO}$, and regulatory authorities, can greatly accelerate early clinical development of promising drug therapies under extraordinary circumstances, such as the SARS-CoV-2 pandemic [26].

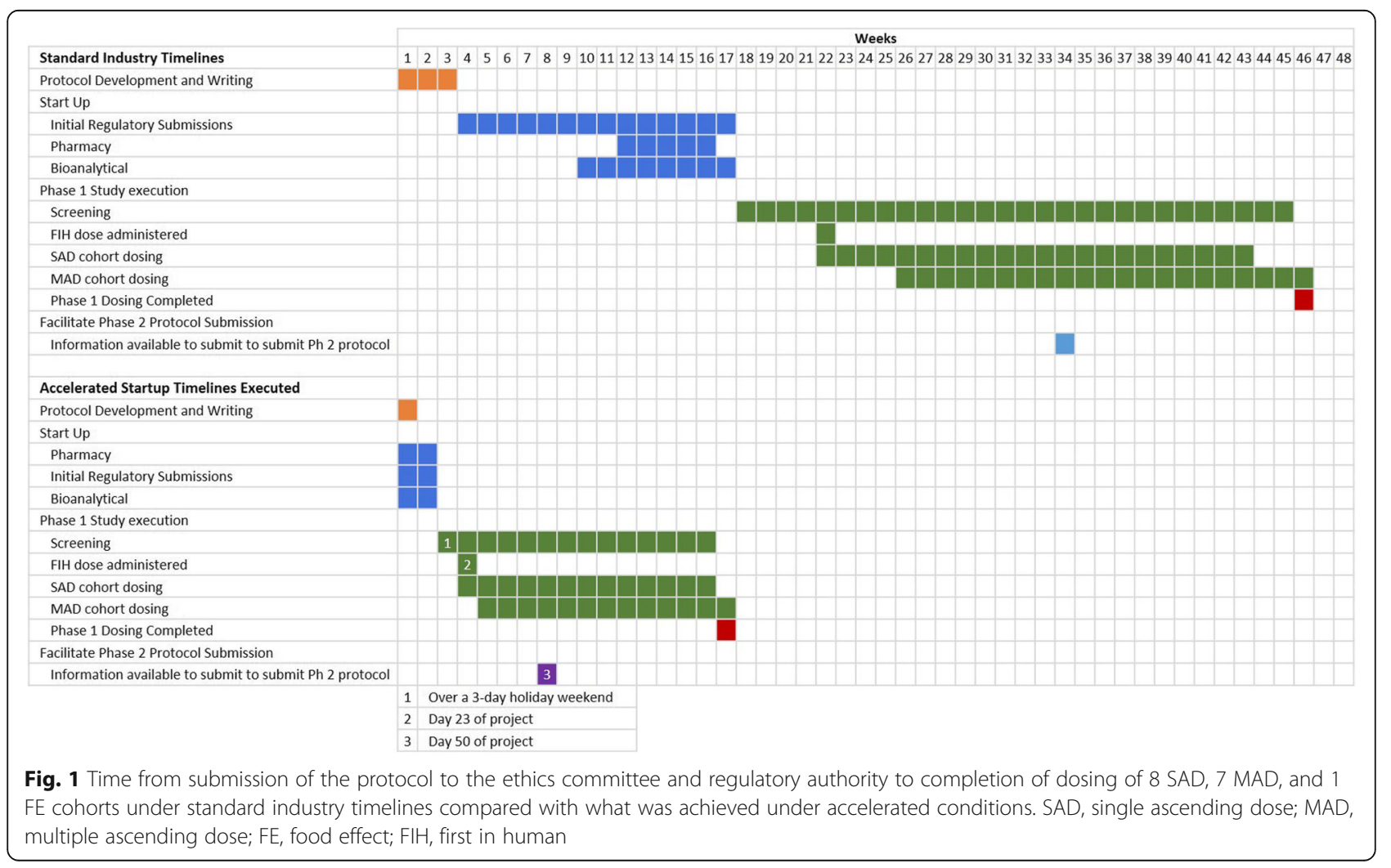




\section{Abbreviations}

API : Active pharmaceutical ingredient; ARDS: Acute respiratory distress syndrome; COVID-19: Coronavirus infectious disease 2019; CRO: Contract research organization; CTA: Clinical trial application; EOS: End of study; FDA: Food and Drug Administration; FE: Food effect; FIH: First-in-human; HRA: Health Research Authority; IAV: Influenza A virus; IND: Investigational new drug; MAD: Multiple ascending dose; MERS-CoV: Middle East respiratory syndrome coronavirus; MHRA: Medicines and Health products Regulatory Authority; MHV: Mouse hepatitis virus; PI: Principal investigator; RdRp: RNAdependent RNA polymerase; REC: Research ethics committee; RSV: Respiratory syncytial virus; SAD: Single ascending dose; SARSCoV: Severe acute respiratory syndrome coronavirus; SARS-CoV-2: Severe acute respiratory syndrome coronavirus 2; UK: United Kingdom; VEEV: Venezuelan equine encephalitis virus

\section{Acknowledgements}

The authors wish to thank the IND team at the Emory Institute for Drug Development (EIDD) and Drug Innovation Ventures at Emory (DRIVE) for the expert and expeditious work, the study participants, and the many individuals who worked tirelessly to accelerate the development of molnupiravir.

\section{Authors' contributions}

All authors were involved in the planning, analysis, and writing of this manuscript.

\section{Funding}

The study was funded in its entirety by Ridgeback Biotherapeutics.

\section{Availability of data and materials}

Refer to clinical trial master file (reference [23]; ClinicalTrials.gov identifier NCT04392219.

\section{Declarations}

\section{Ethics approval and consent to participate}

Refer to reference [23], ClinicalTrials.gov identifier NCT04392219.

\section{Consent for publication}

Not applicable.

\section{Competing interests}

Wendy Holman, Wayne Homan, Stacy McIntosh, and Wendy Painter are employees of Ridgeback Biotherapeutics, which funded the study. George Painter holds a number of patents that are relevant to EIDD-2801 (molnupiravir) - see separately submitted ICMJE form. Jim Bush and Oren Cohen are employees of Covance, the CRO chosen by Ridgeback to assist in the execution of the study described.

\section{Author details}

${ }^{1}$ Ridgeback Biotherapeutics, 3480 Main Highway, Unit 402, Miami, FL 33133, USA. ${ }^{2}$ Emory University School of Medicine, Drug Innovations at Emory (DRIVE) and Emory Institute of Drug Development (EIDD), 954 Gatewood Road, Atlanta, GA 30329, USA. ${ }^{3}$ Covance Clinical Research Unit Ltd., Springfield House, Hyde Street, Leeds LS2 9LH, UK. ${ }^{4}$ Covance, Inc., 6 Drive, Research Triangle Park, Moore, NC 27709, USA.

Received: 28 April 2021 Accepted: 9 August 2021

Published online: 23 August 2021

\section{References}

1. Chan JF, Yuan S, Kok KH, To KKW, Chu H, Yang J, et al. A familial cluster of pneumonia associated with the 2019 novel coronavirus indicating personto-person transmission: a study of a family cluster. Lancet. 2020;395(10223) 514-23. https://doi.org/10.1016/S0140-6736(20)30154-9.

2. Chen N, Zhou M, Dong X, Qu J, Gong F, Han Y, et al. Epidemiological and clinical characteristics of 99 cases of 2019 novel coronavirus pneumonia in Wuhan, China: a descriptive study. Lancet. 2020;39:507-13.

3. Li Q, Guan X, Wu P, Wang X, Zhou L, Tong Y, et al. Early transmission dynamics in Wuhan, China, of novel coronavirus-infected pneumonia. N Engl J Med. 2020;382(13):1199-207. https://doi.org/10.1056/NEJMoa2001316.
4. Wang D, Hu B, Hu C, Zhu F, Liu X, Zhang J, et al. Clinical characteristics of 138 hospitalized patients with 2019 novel coronavirus-infected pneumonia in Wuhan. China. JAMA. 2020;323(11):1061-9. https://doi.org/10.1001/jama.2 020.1585 .

5. Wu F, Zhao S, Yu B, Chen YM, Wang W, Song ZG, et al. A new coronavirus associated with human respiratory disease in China. Nature. 2020;579(7798): 265-9. https://doi.org/10.1038/s41586-020-2008-3.

6. Zhou P, Yang XL, Wang XG, Hu B, Zhang L, Zhang W, et al. A pneumonia outbreak associated with a new coronavirus of probable bat origin. Nature. 2020;579(7798):270-3. https://doi.org/10.1038/s41586-020-2012-7.

7. Zhu N, Zhang D, Wang W, Li X, Yang B, Song J, et al. A novel coronavirus from patients with pneumonia in China, 2019. N Engl J Med. 2020;382(8): 727-33. https://doi.org/10.1056/NEJMoa2001017.

8. Guan W, Ni Z, Hu Y, Liang W, Ou C, He J, et al. Clinical characteristics of coronavirus disease 2019 in China. N Engl J Med. 2020;382(18):1708-20. https://doi.org/10.1056/NEJMoa2002032.

9. Huang C, Wang Y, Li X, Ren L, Zhao J, Hu Y, et al. Clinical features of patients infected with 2019 novel coronavirus in Wuhan. China. Lancet. 2020;395(10223):497-506. https://doi.org/10.1016/S0140-6736(20)30183-5.

10. Vetter P, Vu D1, L'Huillier AG, Schibler M, Kaiser L, Jacquerioz F. Clinical features of COVID-19. BMJ. 2020:369:m1470.

11. Lescure FX, Bouadma L, Nguyen D, Parisey M, Wicky PH, Behillil S, et al. Clinical and virological data of the first cases of COVID-19 in Europe: a case series. Lancet Infect Dis. 2020;20(6):697-706. https://doi.org/10.1016/S1473-3 099(20)30200-0.

12. Zhou F, Yu T, Du R, Fan G, Liu Y, Liu Z, et al. Clinical course and risk factors for mortality of adult inpatients with COVID-19 in Wuhan, China: a retrospective cohort study. Lancet. 2020;395(10229):1054-62. https://doi. org/10.1016/S0140-6736(20)30566-3.

13. Hernandez-Santiago BI, Beltran T, Stuyver L, Chu CK, Schinazi RF. Metabolism of the anti-hepatitis C virus nucleoside beta-D-N4hydroxycytidine in different liver cells. Antimicrob Agents Chemother. 2004; 48(12):4636-42. https://doi.org/10.1128/AAC.48.12.4636-4642.2004.

14. Painter GR, Bowen RA, Bluemling GR, DeBergh J, Edpuganti V, Gruddanti PR, et al. The prophylactic and therapeutic activity of a broadly active ribonucleoside analog in a murine model of intranasal Venezuelan equine encephalitis virus infection. Antiviral Res. 2019;171:104597. https://doi.org/1 0.1016/j.antiviral.2019.104597.

15. Agostini ML, Pruijssers AJ, Chappell JD, Gribble J, Lu X, Andres EL, et al. Small-molecule antiviral $\beta$-d-N4-hydroxycytidine inhibits a proofreadingintact coronavirus with a high genetic barrier to resistance. J Virol. 2019;93: e01348-19.

16. Reynard O, Nguyen XN, Alazard-Dany N, Barateau V, Cimarelli A, Volchkov VE. Identification of a new ribonucleoside inhibitor of Ebola virus replication. Viruses. 2015;7(12):6233-40. https://doi.org/10.3390/v7122934.

17. Toots M, Yoon JJ, Hart M, Natchus MG, Painter GR, Plemper RK. Quantitative efficacy paradigms of the influenza clinical drug candidate EIDD-2801 in the ferret model. Transl Res. 2020;218:16-28. https://doi.org/10.1016/j.trsl.201 9.12.002.

18. Urakova N, Kuznetsova V, Crossman DK, Sokratian A, Guthrie DB, Kolykhalov AA, et al. $\beta$-d-N4-Hydroxycytidine is a potent anti-alphavirus compound that induces a high level of mutations in the viral genome. J Virol. 2018;92: e01965-17.

19. Flavell RA, Sabo DL, Bandle EF, Weissmann C. Site-directed mutagenesis: generation of an extracistronic mutation in bacteriophage Q beta RNA. J Mol Biol. 1974;89(2):255-72. https://doi.org/10.1016/0022-2836(74)90517-8.

20. Mullins JI, Heath L, Hughes JP, Kicha J, Styrchak S, Wong KG, et al. Mutation of HIV-1 genomes in a clinical population treated with the mutagenic nucleoside KP1461. PLoS One. 2011;6(1):e15135. https://doi.org/10.1371/ journal.pone.0015135.

21. Toots M, Yoon JJ, Cox RM, Hart M, Sticher ZM, Makhsous N, et al. Characterization of orally efficacious influenza drug with high resistance barrier in ferrets and human airway epithelia. Sci Transl Med. 2019;11: eaax5866.

22. Sheahan TP, Sims AC, Zhou S, Graham RL, Pruijssers AJ, Agostini ML, et al. An orally bioavailable broad-spectrum antiviral inhibits SARS-CoV-2 in human airway epithelial cell cultures and multiple coronaviruses in mice. Sc Transl Med. 2020;12:eabb5883.

23. Agostini ML, Andres EL, Sims AC, RI G, Sheahan TP, Lu X, et al. Coronavirus susceptibility to the antiviral remdesivir (GS-5734) is mediated by the viral polymerase and the proofreading exoribonuclease. mBio. 2018;9:e00221-18. 
24. Painter WP, Holman W, Bush JA, Almazedi F, Malik M, Eraut NCJE, et al. Human safety, tolerability, and pharmacokinetics of molnupiravir, a novel broad-spectrum oral antiviral agent with activity against SARS-CoV-2. Antimicrob Agents Chem. 2021;65(5). https://doi.org/10.1128/AAC.02428-20.

25. Medicines and Healthcare products Regulatory Agency (MHRA). Guidance: Clinical trials applications for Coronavirus (COVID-19). https://www.gov.uk/ guidance/clinical-trials-applications-for-coronavirus-covid-19 (https://www. gov.uk/guidance/clinical-trials-applications-for-coronavirus-covid-19 published 19 March, 2020).

26. MacKenzie R, Honig P, Sewards J, Goodwin R, Hellio MP. CoVID-19 must catalyse changes to clinical development. Nat Rev Drug Disc. 2020;19(10): 653-4. https://doi.org/10.1038/d41573-020-00149-2.

\section{Publisher's Note}

Springer Nature remains neutral with regard to jurisdictional claims in published maps and institutional affiliations.

Ready to submit your research? Choose BMC and benefit from:

- fast, convenient online submission

- thorough peer review by experienced researchers in your field

- rapid publication on acceptance

- support for research data, including large and complex data types

- gold Open Access which fosters wider collaboration and increased citations

- maximum visibility for your research: over $100 \mathrm{M}$ website views per year

At $\mathrm{BMC}$, research is always in progress.

Learn more biomedcentral.com/submissions 